\title{
Tsafon
}

Revue d'études juives du Nord

$82 \mid 2021$

Enjeux esthétiques dans la littérature après Auschwitz

\section{Tsafon tourne la page}

\section{Emmanuel Persyn}

\section{(2) OpenEdition}

Journals

Édition électronique

URL : https://journals.openedition.org/tsafon/4455

DOI : 10.4000/tsafon.4455

ISSN : 2609-6420

\section{Éditeur}

Association Jean-Marie Delmaire

\section{Édition imprimée}

Date de publication : 1 décembre 2021

Pagination : 133-140

ISSN : $1149-6630$

\section{Référence électronique}

Emmanuel Persyn, «Tsafon tourne la page », Tsafon [En ligne], 82 | 2021, mis en ligne le 01 décembre 2021, consulté le 12 février 2022. URL : http://journals.openedition.org/tsafon/4455 ; DOI : https:// doi.org/10.4000/tsafon.4455 


\section{Édition}

\section{Tsafon tourne la page}

\section{Emmanuel Persyn*}

Les démarches avec le service de valorisation de la recherche de l'université de Lille, annoncées dans des numéros précédents, aboutissent et dès 2022 la revue sera confectionnée par ce service mais les comités de rédaction et scientifique restent inchangés. Au moment où il tourne une page en confiant la mise en page et la diffusion de Tsafon, à l'Université de Lille, seule revue d'études juives de province, le comité éditorial se devait de rendre hommage à la ténacité, à la rigueur et à la compétence de ses fondateurs.

\section{Évolution de la revue}

Lorsqu'ils créent la revue en 1989 en s'appuyant sur la section d'hébreu de l'université de Lille 3, Jean-Marie et Danielle Delmaire, accompagnés de Bernard Meslet, se lancent dans une aventure aussi exaltante que délicate. Il leur faut en effet toute leur énergie et leur attachement aux mondes juifs pour élaborer, définir et réaliser ce projet éditorial. Entreprise artisanale que celle-là comme le pressentait le comité de rédaction: "Nous faisons ici un pari sur l'intelligence et le désir d'apprendre du public, l'aide bénévole pour la frappe et l'illustration, sur l'activité des chercheurs. C'est un pari que nous faisons avec confiance ${ }^{1}$. Il en fallait pour coordonner les bonnes volontés, obtenir à temps les contributions et fabriquer chaque numéro tel un artisan.

\footnotetext{
* Journaliste indépendant, un des premiers membres du comité de rédaction de Tsafon.

${ }^{1}$ Tsafon, revue d'études juives du Nord, $\mathrm{n}^{\circ} 1$, février 1990, p. 3.
} 
Jean-Marie et Danielle Delmaire se sont réjouis des progrès de la technologie : tout juste acquéreurs d'un ordinateur personnel, en 1989, ils se sont lancés dans l'aventure. Ils ont ainsi assuré avec constance, rigueur et compétence la publication et la livraison régulières de cette revue. Des tâches répétitives, fastidieuses et particulièrement ingrates, prises sur le temps précieux de la recherche et de la création. En ont-ils passé des soirées, quelques nuits même à faire de Tsafon une revue de qualité !

Ils souhaitaient faire découvrir ou approfondir le judaïsme et l'hébreu. C'est bien ce que le comité de rédaction, alors constitué de neuf autres personnes en plus d'eux - universitaires, enseignants d'hébreu, «personnes connues pour animer souvent les activités culturelles de la communauté juive de Lille $»^{2}$ notamment - explique dans l'éditorial du premier numéro en février 1990 : « Le développement des études juives et hébraïques dans notre région et l'intérêt d'un public croissant envers ces études nous ont incité à franchir une étape importante en vous présentant [Tsafon] $»^{3}$, la revue d'études juives du Nord.

Jean-Marie et Danielle Delmaire ont tout d'abord repéré des auteurs - biblistes, linguistes, historiens, philosophes... - de tous pays - Allemagne, Israël, Belgique... - et obtenu leur concours. La revue est progressivement devenue une référence internationale. De nombreuses bibliothèques y sont, par exemple, abonnées.

Le désir de rendre compte de la recherche la plus pointue dans les domaines des études juives s'accompagnait de celui de faire connaître les travaux des plus jeunes. Excellents pédagogues, Jean-Marie et Danielle Delmaire tenaient également à publier les travaux d'étudiants et de jeunes collègues. Les numéros $22-23$ et 32 , pour ne prendre que ceux-là, réunissent par exemple les travaux des étudiants de la section d'hébreu. Ils étaient ensuite très attachés à rendre accessibles ces connaissances au plus grand nombre, aux chercheurs bien sûr mais aussi au grand public. « Notre revue, écrivent-ils dans le premier éditorial, ne sera pas que réservée à ces recherches universitaires, qui tiendront cependant une place essentielle. Elle ouvre ses pages à des essais, des résumés de conférences, des expressions culturelles diverses des diverses communautés juives du Nord et de Belgique $»^{4}$.

Dans la première décennie de son existence, la revue publie trimestriellement des articles divers, quelques numéros seulement

\footnotetext{
${ }^{2}$ Ibid., p. 1.

${ }^{3}$ Ibid.

${ }^{4}$ Ibid., p. 2.
} 
présentent un corpus thématique. La revue se distingue aussi par la publication dans chaque numéro d' « un grand texte inédit en français ». Et de préciser, toujours dans ce premier numéro : «textes historiques ou littéraires traduits de l'hébreu ou langue juive, parfois en présentation bilingue quand nous obtiendrons l'autorisation nécessaire des éditeurs $\rangle^{5}$. Dès le premier numéro, la revue livre des recensions d'ouvrages et des informations sur les études juives ou des manifestations scientifiques.

À la mort de Jean-Marie Delmaire, voici déjà près d'un quart de siècle, Danielle Delmaire a poursuivi la publication de Tsafon dans le même esprit après la constitution, en 1998, de l'association Jean-Marie Delmaire. Ce faisant, le comité de rédaction prenait la décision de se séparer de la section d'hébreu de l'université de Lille 3 puisqu'aucun de ses membres n'appartenait à cette structure. En même temps, décision était prise de semestrialiser la revue. Au total sur une année, le nombre de pages restait d'environ 400 mais les frais de réalisation et d'envoi étaient réduits. Désormais depuis le $\mathrm{n}^{\circ} 37$ de juin 1999, Tsafon présente un dossier thématique, un ou deux articles divers (Varia), l'édition d'un document d'archives ou la traduction d'un texte en hébreu, et comme auparavant les dernières pages sont occupées par des recensions d'ouvrages, des informations et parfois un hommage à des personnalités ou des amis de la revue.

La fin de la décennie 2000, voit la création du site de Tsafon où apparaissent les sommaires de tous les numéros. Depuis 2016, bénéficiant toujours des progrès techniques, la revue paraît en lecture libre sur le site openedition.org. Cette parution a été facilitée grâce à l'université de Lille 3 que la revue a pu réintégrer pour être désormais une publication universitaire.

Enfin depuis 2003, le comité de rédaction s'est lancé dans la publication de numéros hors-série centrés sur un thème unique mais trop abondant pour être réduit à la centaine de pages d'un dossier d'un numéro semestriel. Ces hors-série paraissent irrégulièrement.

\section{Les comités et les abonnés}

Le comité de rédaction du premier numéro était dirigé conjointement par Jean-Marie Delmaire et Matty Cohen, tandis que Bernard Meslet assurait le secrétariat. Le décès prématuré de ce dernier et le départ de Matty Cohen, dans les mois qui suivaient la création de la

${ }^{5}$ Ibid., p. 3. 
revue, laissaient Jean-Marie Delmaire seul à la direction. Puis ce fut son décès qui occasionna une nouvelle organisation avec Danielle Delmaire comme directrice de publication. Actuellement elle est assistée par un comité de rédaction étoffé qui passe de cinq membres au seuil des années 2000 à aujourd'hui 13 membres en plus d'elle et par un comité scientifique qui réunit 16 personnes.

La comparaison du sommaire du premier numéro et du numéro 82 est très significative. Elle montre bien la permanence des orientations initiales malgré les évolutions que traduisent l'augmentation et la diversification des contributeurs, la mise en œuvre d'une véritable maquette, le recours à la couleur, etc.

Durant ces 32 ans, 82 numéros ont été publiés et lus par plusieurs centaines d'abonnés, dont des institutions aussi prestigieuses que les bibliothèques Sainte Geneviève, de Alliance israélite universelle, du Mémorial de la Shoah et la Bibliothèque Universitaire des Langues et Civilisations, pour Paris ; la Bibliothèque nationale et l'université Bar Ilan pour Israël ; les universités de Los Angeles (UCLA), Cornell University de New York, Yale University de New Haven, Klau library de Cincinnati, Hebrew Union College et New York Public Library, pour les Étas-Unis ; Universitaetsbibliothek Johann Christian Senckenberg de Frankfurt et l'université de Tübingen pour l'Allemagne.

Quant au nombre des abonnés il n'a cessé de progresser durant la première décennie pour atteindre plus de la centaine dans les années 2000. Avec la lecture gratuite en ligne, ce nombre a diminué passant à 80 puis à 60 en 2020. Les lecteurs en ligne peuvent toujours adhérer à l'association Jean-Marie Delmaire qui compte depuis sa création une cinquantaine de fidèles.

\section{Les thèmes abordés}

En parcourant le sommaire des 82 numéros publiés jusqu'à aujourd'hui et des neuf hors-série, le lecteur ne peut qu'être étonné par la diversité des sujets traités: Bible, linguistique, littérature, histoire, droit, philosophie, art...

Entre autres numéros, on retiendra le numéro double 6-7 (décembre 1991) à l'occasion du centenaire de la synagogue de Lille, le $n^{\circ} 14-15$ sur les accords d'Oslo (décembre 1993), le n 16 sur l'affaire Dreyfus (hiver 1994 à l'occasion du centenaire du début de l'Affaire) ou encore le ${ }^{\circ} 33$ 34 sur la littérature israélienne (juin 1998). Puis lorsque la revue se 
restructure en offrant à lire un dossier, on peut rassembler plusieurs thèmes dans l'ensemble de la production.

- Les Juifs dans la Seconde Guerre mondiale : un thème qui avait déjà retenu l'attention du comité de rédaction avec les $n^{\circ} 9-10$ et 21 qui livrent les listes des déportés juifs du Nord et du Pas-de-Calais après leur découverte, dans des archives belges, par Danielle Delmaire; thème complété par les $n^{\circ} 47$ (juin 2004) : être jeune juif pendant la Seconde Guerre mondiale, 73 (juin 2017): enquête sur la réception de l'enseignement de la Shoah et 79 (juin 2020) sur la particularité du sort des Juifs dans la région rattachée au gouvernement militaire allemand de Bruxelles. D'autres numéros ont publié dans la rubrique «Varia » les souvenirs de rescapés de la Shoah.

- Les études bibliques : un premier numéro, 38 (décembre 1999), présente un dossier sur Jérusalem dans le temps et l'espace, dans lequel des références bibliques apparaissaient; le dossier du $n^{\circ} 40$ (décembre 2000) porte essentiellement sur l'étude de la Bible hébraïque tandis que le dossier du $\mathrm{n}^{\circ} 57$ (juin 2009) est entièrement consacré au Cantique des Cantiques, que le thème du dossier du $n^{\circ} 58$ (décembre 2009) porte sur le Talmud et que le dossier du $n^{\circ} 77$ (juin 2019) offre des études sur les traductions de la Bible.

- La littérature : c'est un thème qui a occupé plusieurs dossiers et qui avait déjà été présenté dans des numéros antérieurs à la présentation de dossiers. Le $n^{\circ} 39$ (juin 2000) évoque la littérature juive d'Europe centrale et orientale; les $n^{\circ} 50$ (décembre 2005) et 66 (décembre 2013) interrogent sur les rapports entre la Bible et la littérature ; le $n^{\circ} 70$ (décembre 2015) étudie les œuvres d'écrivains juifs de langue française et le dossier du ${ }^{\circ}$ 72 (décembre 2016) a pour thème : Juifs et Israéliens dans la littérature française et israélienne. La poésie hébraïque moderne n'est pas oubliée grâce au n 59 (juin 2010).

- Les langues et l'hébreu: le dossier du $n^{\circ} 45$ (juin 2003) questionne sur 1'hébreu : est-il une langue morte, ressuscitée et vivante ? Et deux autres dossiers des $n^{\circ} 49$ (juin 2005) et 75 (juin 2018) portent sur les rapports entre les langues et l'exil, respectivement la « langue et culture d'exil » et l'« exil des langues et langues d'exil ».

- La vie juive dans différentes villes ou pays : là encore, un numéro antérieur aux dossiers ( $\mathrm{n}^{\circ}$ double 26-27 de 1996) est consacré à la ville de Safed pour conforter le jumelage de la ville israélienne avec Lille où est implantée la revue ; il est suivi par une série de numéros qui contiennent des dossiers sur la vie juive en Allemagne $n^{\circ} 42$ (décembre 2001), en 
Autriche $n^{\circ} 46$ (décembre 2003), en Italie $n^{\circ} 48$ (décembre 2004) et à Prague ( $\mathrm{n}^{\circ} 52$ décembre 2006). Enfin le dossier du n 60 (décembre 2010) rapporte « 1000 ans de vie juive en Pologne ».

- Des années d'anniversaire ont suscité des dossiers : c'est déjà le cas avec le $\mathrm{n}^{\circ} 16$ à propos du centenaire de l'affaire Dreyfus en 1994. L'idée est reprise pour présenter la situation en Israël « Dix ans après les accords d'Oslo » dans le $n^{\circ} 44$ (décembre 2002), pour commémorer les débuts de Tel Aviv dans le $n^{\circ} 55$ (juin 2008) et l'engagement des Juifs dans la Grande Guerre. Pour ce thème, Tsafon bénéficia de nombreuses collaborations d'auteurs qu'il fallut répartir sur les deux n ${ }^{\circ} 67$ et 68 (juin et décembre 2014). Le souvenir de l'expulsion des Juifs d'Égypte, suite à la guerre du Sinaï, est présent dans le dossier du $n^{\circ} 71$ (juin 2016) tandis que celui de la Déclaration Balfour occupe le dossier du n ${ }^{\circ} 74$ (décembre 2017).

- Un numéro accorde un hommage particulier à Jean-Marie Delmaire, à l'occasion des quinze premières années d'existence de la revue et de son cinquantième numéro (décembre 2005).

Bien d'autres Varia ou éditions ont également participé à la connaissance de ces divers thèmes.

Quant aux thèmes des neuf numéros hors-série, ils se répartissent comme suit: une chronologie biblique pour le premier numéro, puis une édition bilingue (yiddish-français et hébreu-français) de Poètes de la Shoah (avril 2007), trois ouvrages sur les Juifs dans la Seconde Guerre mondiale dans la région nord: le judenlager des Mazures dans les Ardennes françaises (octobre 2007), les persécutions à l'encontre des Juifs et des Tsiganes dans le Douaisis (octobre 2008), la spoliation des Juifs et des Tsiganes dans la «Zone rattachée » et en Belgique, numéro qui rassemble les actes d'une journée d'études (novembre 2012). Le $n^{\circ} 6$ (octobre 2013) est un épais volume sur les Juifs d'Algérie. Le centenaire de la naissance de l'historien Bernhard Blumenkranz est l'occasion de lui consacrer le $\mathrm{n}^{\circ} 7$ (octobre 2014). Et de nouveau une journée d'études permet d'élaborer le $n^{\circ} 8$ (novembre 2015) sur les « représentations juives du christianisme, $\mathrm{XIX}^{\mathrm{e}}$ et $\mathrm{XX}^{\mathrm{e}}$ siècles ». Enfin, dans le $n^{\circ} 9$ (mars 2018), Tsafon a entendu la voix d'une rescapée de la Shoah, Asia Turgel qui vécut à Wilno, connut les ghettos et la déportation.

En tournant ainsi la page, Tsafon se donne les moyens humains et matériels de sa pérennité avec la même orientation d'ouverture, de tolérance et de rigueur scientifique qu'à sa création. 
Trente années de Tsafon

Quelques premières pages de couverture

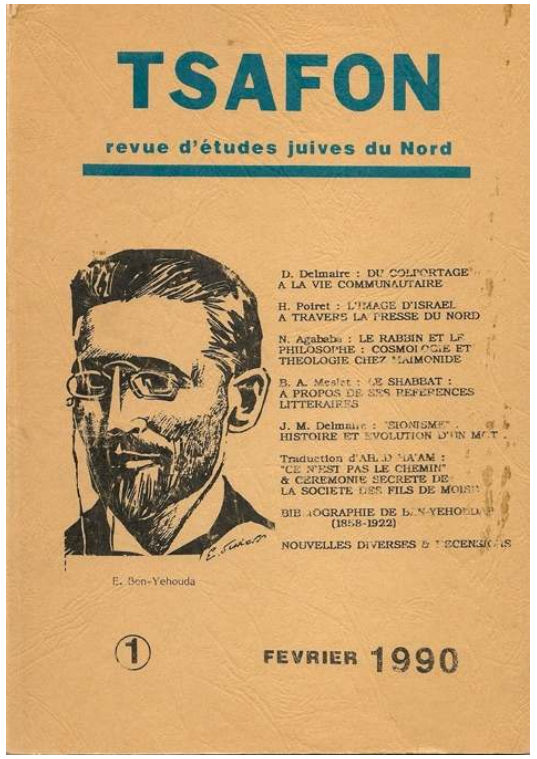

TSAFON revue d'études juives du Nord n 6-7

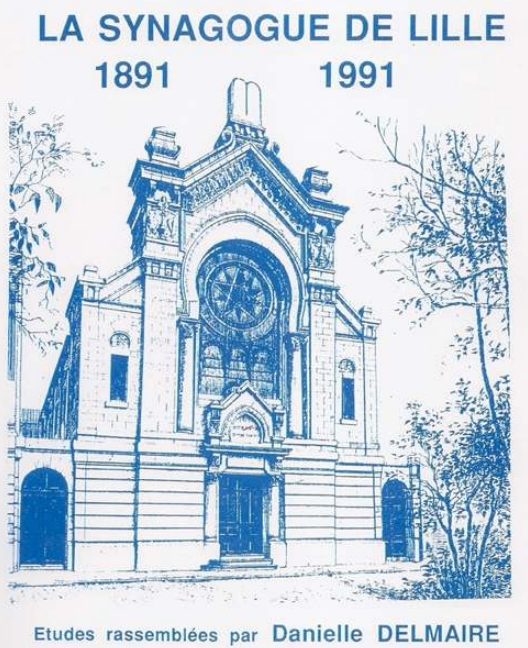

T S A F O N

Revue d'études juives du Nord
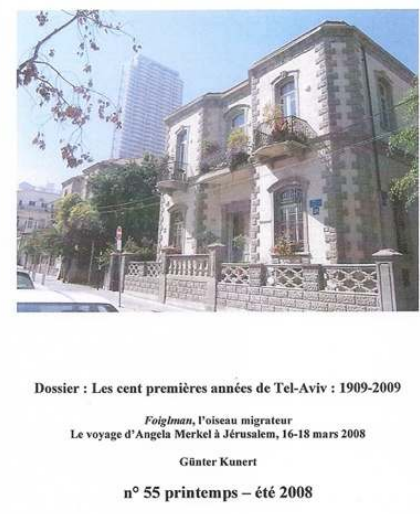

\section{T S A F O N}

Revue d'études juives du Nord
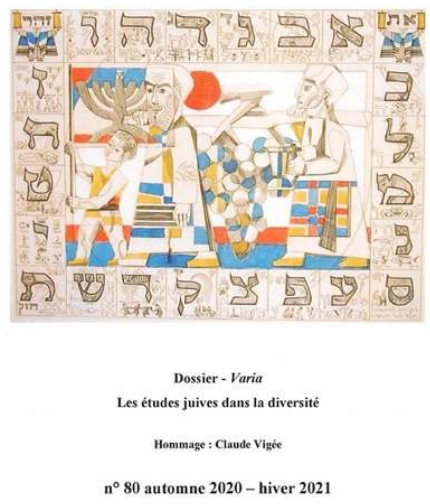
Quinze années de Tsafon

Couvertures du $\mathrm{n}^{\circ} 50$ (décembre 2005)

\section{T S A F O N}

Revue d'études juives du Nord

\section{Tsafon}

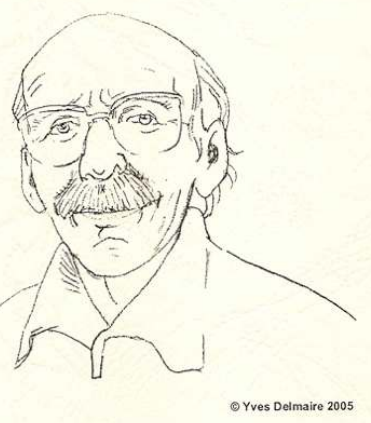

Page une de couverture du $\mathrm{n}^{\circ} 50$ En hommage à son fondateur Jean-Marie Delmaire (caricature d'Yves Delmaire)

Hommage à Jean-Marie Delmaire

$\mathrm{n}^{\circ} \mathbf{5 0}$ automne 2005 - hiver 2006

\section{Tsafon}

Page quatre de couverture du $n^{\circ} 50$ (caricature d'Yves Delmaire)

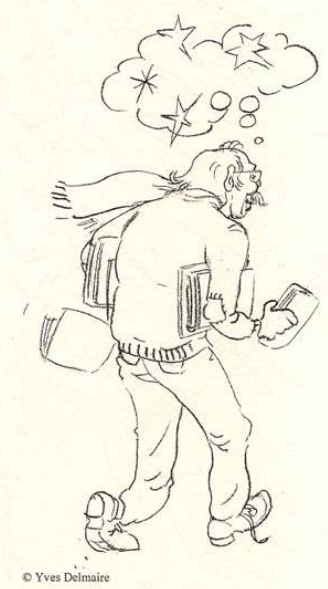

